\title{
The influence of frailty under direct oral anticoagulant use in patients with atrial fibrillation
}

\author{
Takashi Yamamoto, ${ }^{1}$ Kentaro Yamashita, ${ }^{1}$ Kiichi Miyamae, ${ }^{1}$ Yuichiro Koyama, ${ }^{1}$ \\ Masataka Izumimoto, ${ }^{1}$ Yoshihiro Kamimura, ${ }^{1}$ Satoko Hayakawa, ${ }^{1}$ Kazutaka Mori, ${ }^{1}$ \\ Takaaki Yamada, ${ }^{1}$ Yasushi Tomita, ${ }^{1}$ Toyoaki Murohara ${ }^{2}$
}

\begin{abstract}
- Additional material is
published online only. To view please visit the journal online (http://dx.doi.org/10.1136/ heartasia-2019-011212).
\end{abstract}

'Department of Cardiology, National Hospital Organization Nagoya Medical Center, Nagoya, Japan

${ }^{2}$ Department of Cardiology, Nagoya University Graduate School of Medicine, Nagoya, Japan

\section{Correspondence to}

Dr Kentaro Yamashita, Cardiology, National Hospital Organization Nagoya Medical Center, Nagoya 460-0001, Japan; yamaken2929@gmail. com

Received 12 March 2019 Revised 17 May 2019

Accepted 23 May 2019

\section{Check for updates}

(C) Author(s) (or their employer(s)) 2019. No commercial re-use. See rights and permissions. Published by BMJ.

To cite: Yamamoto $T$, Yamashita K, Miyamae K, et al. Heart Asia

2019:11:e011212

doi:10.1136/

heartasia-2019-011212

\section{ABSTRACT}

Background Frailty is a prognostic factor in patients with atrial fibrillation (AF). However, there is no report on the associations between frailty and clinical adverse events in patients with AF taking direct oral anticoagulants (DOAC). The factors related to the occurrence of clinical adverse events are still under discussion. Therefore, we examined the associations between frailty and clinical adverse events in patients with AF taking DOAC in daily clinical practice.

Methods We retrospectively evaluated 240 consecutive patients with AF who had been newly prescribed DOAC in our hospital from April 2016 through May 2017. Data collected included Clinical Frailty Scale (CFS) scores, laboratory results and basic demographic information. Results During the mean follow-up period of 13.4 months, 20 patients died (7.6 per 100 person-years), stroke or systemic embolism occurred in seven patients (2.6 per 100 person-years) and major bleeding occurred in 11 patients (4.2 per 100 person-years). We defined these adverse events as composite end points, and we estimated adjusted $\mathrm{HRs}$ and $95 \% \mathrm{Cls}$ for risk factors using the Cox proportional hazard regression model. Frailty (defined as a CFS score of 5 or more; HR: 3.71; 95\% Cl: 1.59 to 8.65 ), female sex (HR: $3.49 ; 95 \% \mathrm{Cl}$ : 1.73 to 7.07$)$, serum albumin level (HR: $0.47 ; 95 \% \mathrm{Cl}$ : 0.28 to 0.79 ) and malignancy (HR: $4.02 ; 95 \% \mathrm{Cl}: 1.83$ to 8.84 ) were independent predictors of the composite end points.

Conclusions Frailty, female sex, hypoalbuminaemia and malignancy were associated with clinical adverse events in patients with $\mathrm{AF}$ who were prescribed DOAC.

\section{INTRODUCTION}

Both the number of elderly people and the prevalence of atrial fibrillation (AF) are increasing worldwide. ${ }^{12}$ Anticoagulation therapy for elderly patients with AF is important because of the high embolic risk in this population. ${ }^{3}$ The efficacy and safety of direct oral anticoagulants (DOAC) in patients with AF have been proven in large-scale clinical trials. ${ }^{45}$ The risk of bleeding or death is increased in elderly patients with $\mathrm{AF}^{6-8}$ In addition, we sometimes observe cases in which clinically serious adverse events (eg, bleeding, stroke and death) occur over a short period of time after DOAC administration. However, it is not appropriate to dichotomise people solely based on age. Therefore, it remains controversial whether DOAC should be prescribed for this group.

In recent years, frailty status has drawn attention as being a strong predictor of mortality in elderly

\section{Key questions}

What is already known about this subject?

- Frailty is a prognostic factor in patients with atrial fibrillation (AF), but the associations between frailty and adverse events in direct oral anticoagulant (DOAC) users are poorly understood.

\section{What does this study add?}

- The incidence of adverse events (ie, all-cause mortality, stroke or systemic embolism and major bleeding) was high in patients with $\mathrm{AF}$ after DOAC administration. Furthermore, these events occurred over a median of 3.6 months (IQR: 1.3-9.3 months) in frail patients. Frailty is significantly associated with the incidence of adverse events than other proven risk factors.

How might this impact on clinical practice?

- In frail patients with AF, more careful followup of adverse events is necessary after DOAC administration.

people. ${ }^{9}$ There have also been reports that AF is associated with frailty. ${ }^{10}$ Frailty is a prognostic factor in patients with $\mathrm{AF},{ }^{11}$ but to our knowledge, there are no reports on the prognostic potential of frailty in patients with AF who are taking DOAC. Therefore, we examined the associations between frailty and clinical adverse events in patients with AF taking DOAC in daily clinical practice.

\section{MATERIALS AND METHODS}

\section{Study patients}

Using the data warehouse and search functions of the electronic medical records (HOPE LifeMark-HX, FUJITSU), we retrospectively evaluated 240 consecutive patients with AF who had been newly prescribed DOAC in our hospital from April 2016 through May 2017.

All patients were screened using the ICD-10 (the 10th revision of the International Statistical Classification of Diseases and Related Health Problems) code I48, and then AF was confirmed by an ECG or Holter ECG. Patients who had previously received anticoagulants including DOAC were excluded.

This study was conducted according to the Declaration of Helsinki and was approved by the Institutional Review Board of Nagoya Medical Centre. 


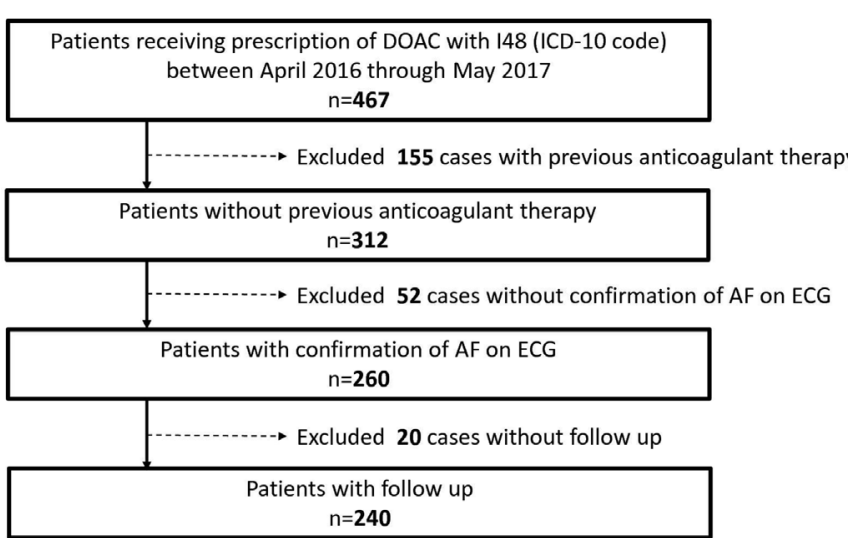

Figure 1 Subject selection. $A F$, atrial fibrillation; $D O A C$, direct oral anticoagulant.

\section{Data collection}

Detailed patient characteristics including age, sex, past medical history, smoking history, blood pressure, body weight, prescription history and laboratory results were collected. To determine the $\mathrm{CHA}_{2} \mathrm{DS}_{2}$-VASc (Cardiac failure or dysfunction, Hypertension, Age $\geq 75$ [Doubled], Diabetes, Stroke[Doubled]-Vascular disease, Age 65-74 and Sex category [Female]) score, points were assigned to each of the following conditions, and the sum was calculated: congestive heart failure, hypertension, age (patients aged $\geq 75$ years received two points and patients aged $65-74$ years received one point), diabetes mellitus, vascular disease, female sex and prior stroke or transient ischaemic attack (TIA; two points). ${ }^{12}$ We included a history of hypertension, rather than blood pressure greater than $160 \mathrm{~mm} \mathrm{Hg}$, because even well-controlled hypertension is an independent risk factor for stroke. ${ }^{13}$ Diabetes mellitus was defined as a fasting plasma glucose concentration $>126 \mathrm{mg} / \mathrm{dL}$ and/or a glycosylated haemoglobin concentration $\geq 6.5 \%$ (National Glycohemoglobin Standardisation Programme), or intake of any antidiabetic agents. Current and ex-smokers were defined as 'smoker'. The estimated glomerular filtration rate (eGFR) was calculated using the equation for Japanese subjects recommended by the Japanese Society of Nephrology: eGFR $\left(\mathrm{mL} / \mathrm{min} / 1.73 \mathrm{~m}^{2}\right)=194 \times \mathrm{SCr}^{-1.094} \times \mathrm{Age}^{-0.287}$ $\times 0.739$ (if female). ${ }^{14}$ Cancer was defined as malignancy diagnosed within the previous 6 months; recurrent, regionally advanced or metastatic cancer; cancer for which treatment had been administered within 6 months prior to DOAC prescription; or haematological cancer that was not in complete remission.

\section{Clinical Frailty Scale}

The Clinical Frailty Scale (CFS) was developed and validated by Rockwood et al. ${ }^{15}$ Patients are assigned a score of 1-9 (1: very fit; 2: well; 3: managing well; 4: vulnerable; 5: mildly frail; 6: moderately frail; 7: severely frail; 8: very severely frail and 9: terminally ill) based on a semiquantitative evaluation of their symptoms, level of inactivity, exhaustion and basic or instrumental activities of daily living. CFS scores were determined by trained physicians based on the records of nurses who directly met with each patient. Patients were classified into one of two categories based on CFS scores: frail (a score of 5-9) or non-frail (a score of 1-4).

\section{Clinical outcomes}

Follow-up patient data were obtained from the medical records on 2 May 2019. We defined all-cause mortality, stroke or systemic embolism (SE) confirmed by imaging, or major bleeding that occurred during the follow-up period as composite end points. Major bleeding was defined as a reduction in haemoglobin by at least $2 \mathrm{~g} / \mathrm{dL}$, transfusion of at least 2 units of blood, or symptomatic bleeding in a critical area or organ using definitions set out by The International Society on Thrombosis and Haemostasis. ${ }^{16}$

\section{Statistical analyses}

Categorical data are presented as percentages (\%), and consecutive patient data are presented as mean \pm SD or median (range), depending on their distribution. We compared categorical variables using the $\chi^{2}$ test when appropriate; otherwise, we used Fisher's exact test. We compared continuous variables using the Student's or Welch's t-test on the basis of the dispersion when appropriate; otherwise, we used the Mann-Whitney U test on the basis of distribution. The incidence rate of each composite end point was calculated as the number per 100 person-years with 95\% CIs. A Cox proportional hazards regression analysis was used to assess the association between factors and composite end points. Any statistically significant covariates (ie, $\mathrm{p}<0.05$ ) in the univariate analysis were included in the multivariate analysis using a downward stepwise regression method. The thresholds for entry and removal were 0.05 and 1.0, respectively. We also analysed the incidence rate of the composite end points in each group stratified by frailty status as determined using CFS scores (frail: 5-9; and non-frail: 1-4), and assessed the difference between them using a log-rank test. All statistical analyses were done using JMP V.9.0 (SAS Institute, Cary, NC, USA), and all tests were two-tailed with the statistical significance set at $\mathrm{p}<0.05$.

\section{RESULTS}

We enrolled 240 patients, as shown in figure 1 . Table 1 shows the baseline characteristics of these patients. The mean age of the patients was $76.1 \pm 10.0$ years and $42.9 \%$ of them were women. The median $\mathrm{CHA}_{2} \mathrm{DS}_{2}$-VASc score was 4 , and the median HAS-BLED (Hypertensioin, Abnormal renal/liver function, Stroke, Bleeding history or predispositioin, Labile international normalization ratio, Elderly (>65 years), Drug/alcohol concomitantly) score was 2 . An under-dose of DOAC was observed in $8.33 \%$ of patients. The median eGFR and serum albumin level were $63.8 \mathrm{~mL} / \mathrm{min} / 1.73 \mathrm{~m}^{2}$ and $3.60 \mathrm{~g} / \mathrm{dL}$, respectively. Frail patients constituted $50.0 \%$ of the total patient population.

The frail group had a higher proportion of women $(55.8 \%$ vs $30.0 \% ; \mathrm{p}<0.001)$, and was older $(81.2 \pm 7.9$ years vs $71.0 \pm 10.0$ years; $\mathrm{p}<0.001)$ and lighter in weight $(52.5 \mathrm{~kg}$ vs $60.5 \mathrm{~kg}$; $\mathrm{p}<0.001)$, compared with the non-frail group. The median CFS (6 vs $3 ; \mathrm{p}<0.001), \mathrm{CHA}_{2} \mathrm{DS}_{2}$-VASc score $(5$ vs $3 ; \mathrm{p}<0.001$ ) and HAS-BLED score (2 vs 1 ; $\mathrm{p}<0.001$ ) were higher in frail group compared with the non-frail group. History of stroke was more common in the frail group $(58.3 \%$ vs $27.5 \%$; $p<0.001)$. Serum albumin $(3.3 \mathrm{~g} / \mathrm{dL}$ vs $4.0 \mathrm{~g} / \mathrm{dL} ; \mathrm{p}<0.001)$ and haemoglobin $(12.3$ $\mathrm{mg} / \mathrm{dL}$ vs $13.7 \mathrm{mg} / \mathrm{dL}$; $\mathrm{p}<0.001)$ levels were lower in the frail group.

During the median follow-up period of 9.2 months (IQR: 1.5-25.5 months), seven patients had a stroke or SE, 11 had a major bleed and 20 died. In total, 37 patients had a composite end point event (14.0 per 100 person-years). The incidence of stroke or SE, major bleeding, all-cause mortality, and stroke, SE or all-cause mortality in the frail group were 6.2 per 100 personyears, 8.3 per 100 person-years, 16.6 per 100 person-years and 30.2 per 100 person-years, respectively (table 2 ).

In the univariate analysis, age, female sex, weight, heart failure, haemoglobin, serum albumin, frailty, malignancy, 
Table 1 Baseline characteristics of the patients

\begin{tabular}{|c|c|c|c|c|}
\hline Characteristics & Total $(n=240)$ & Frail $(n=120)$ & Non-frail $(n=120)$ & P value* \\
\hline Female, $\mathrm{n}(\%)$ & $103(42.9)$ & $67(55.8)$ & $36(30.0)$ & $<0.001$ \\
\hline Age, years & $76.1 \pm 10$ & $81.2 \pm 7.8$ & $71.0 \pm 10$ & $<0.001$ \\
\hline Weight, kg & $57.0(48.4-64.9)$ & $52.5(44.3-60.0)$ & $60.5(53.5-67.0)$ & $<0.001$ \\
\hline CFS & $4.5(3.0-6.0)$ & $6.0(6.0-7.0)$ & $3.0(3.0-4.0)$ & $<0.001$ \\
\hline Hypertension, $\mathrm{n}(\%)$ & $147(61.3)$ & $78(65.0)$ & $69(57.5)$ & 0.233 \\
\hline Diabetes mellitus, n (\%) & $42(17.5)$ & $22(18.3)$ & $20(16.7)$ & 0.734 \\
\hline Smoking, $\mathrm{n}(\%)$ & $118(49.2)$ & $49(40.8)$ & $69(57.5)$ & 0.010 \\
\hline Heart failure, n (\%) & $101(42.1)$ & $53(44.2)$ & $48(40.0)$ & 0.513 \\
\hline Bleeding history, $\mathrm{n}(\%)$ & $14(5.8)$ & $9(7.5)$ & $5(4.2)$ & 0.271 \\
\hline Stroke/TIA, n (\%) & $103(42.9)$ & $70(58.3)$ & $33(27.5)$ & $<0.001$ \\
\hline Paroxysmal AF, n (\%) & $126(52.9)$ & $56(46.7)$ & $70(58.3)$ & 0.070 \\
\hline $\mathrm{CHA}_{2} \mathrm{DS}_{2}$-VASc score & $4(3-5)$ & $5(4-6)$ & $3(2-4)$ & $<0.001$ \\
\hline HAS-BLED score & $2(1-2)$ & $2(1-2)$ & $1(1-2)$ & $<0.001$ \\
\hline BUN (mg/dL) & $17(13-21)$ & $17(12-21)$ & $16(13-20)$ & 0.855 \\
\hline $\mathrm{eGFR}\left(\mathrm{mL} / \mathrm{min} / \mathrm{m}^{2}\right)$ & $63.8(51.7-78.1)$ & $67.0(51.8-82.6)$ & $62.7(51.7-72.0)$ & 0.124 \\
\hline Albumin (g/dL) & $3.6(3.1-4.0)$ & $3.3(2.7-3.7)$ & $4.0(3.5-4.2)$ & $<0.001$ \\
\hline $\mathrm{Hb}(\mathrm{mg} / \mathrm{dL})$ & $12.9(11.2-14.2)$ & $12.3(10.7-13.3)$ & $13.7(12.2-14.7)$ & $<0.001$ \\
\hline DOAC under-dosed (\%) & $20(8.3)$ & $8(6.7)$ & $12(10)$ & 0.350 \\
\hline DOAC over-dosed (\%) & $13(5.4)$ & $5(4.2)$ & $8(6.7)$ & 0.392 \\
\hline$\beta$-blocker, n (\%) & $118(49.2)$ & $56(46.7)$ & $62(51.7)$ & 0.439 \\
\hline ACE-I/ARB, n (\%) & $87(36.3)$ & $38(31.7)$ & $49(40.8)$ & 0.140 \\
\hline Mineralocorticoid receptor antagonist, n (\%) & $37(15.4)$ & $23(19.2)$ & $14(11.7)$ & 0.108 \\
\hline Antiplatelet drugs, n (\%) & 39 (16.3) & $19(15.8)$ & $20(16.7)$ & 0.861 \\
\hline Malignancy, n (\%) & $20(8.3)$ & $13(10.8)$ & $7(5.8)$ & 0.161 \\
\hline
\end{tabular}

Values are presented as mean \pm SD, median (IQR) or $n(\%)$.

${ }^{*} \mathrm{P}$ value for comparison between frail and non-frail from log-rank test.

ACE-I, ACE inhibitor; AF, atrial fibrillation; ARB, angiotensin II receptor blocker; BUN, blood urea nitrogen; CFS, Clinical Frailty Scale; DOAC, direct oral anticoagulants; TIA, transient ischaemic attack; eGFR, estimated glomerular filtration rate.

$\mathrm{CHA}_{2} \mathrm{DS}_{2}$-VASc score and HAS-BLED score were significantly associated with the composite end points. In the multivariate analysis, frailty (HR: $3.71,95 \% \mathrm{CI}: 1.59$ to 8.65 ), female sex (HR: 3.49 , 95\% CI: 1.73 to 7.07 ), serum albumin level (HR: $0.47,95 \% \mathrm{CI}: 0.28$ to 0.79 ) and malignancy (HR: $4.02,95 \%$ CI: 1.83 to 8.84 ) were confirmed as statistically significant factors associated with an increased risk of the composite end points (table 3). The Kaplan-Meier curves stratified by frailty category are shown in figure 2 . The cumulative incidence rate was significantly higher in the frail group compared with the non-frail group (log-rank test; $\mathrm{p}<0.001$ ).

Table 2 Incidence of clinical events during follow-up (per 100 person-years)

\begin{tabular}{llllc}
\hline & $\begin{array}{l}\text { Total } \\
(\mathbf{n}=240)\end{array}$ & $\begin{array}{l}\text { Frail } \\
(\mathrm{n}=120)\end{array}$ & $\begin{array}{l}\text { Non-frail } \\
(\mathrm{n}=120)\end{array}$ & P value* $^{*}$ \\
\hline $\begin{array}{l}\text { Stroke/SE/Major bleeding /all-cause } \\
\text { mortality }\end{array}$ & 14.0 & 30.2 & 4.8 & $<0.001$ \\
$\begin{array}{l}\text { Stroke/SE } \\
\text { Major bleeding }\end{array}$ & 2.6 & 6.2 & 0.6 & 0.024 \\
All-cause death & 4.2 & 8.3 & 1.8 & 0.038 \\
\hline \multicolumn{1}{c}{ Cardiac death } & 7.6 & 16.6 & 2.4 & $<0.001$ \\
\hline \multicolumn{1}{c}{ Non-cardiac death } & 1.5 & 2.1 & 1.2 & 0.583 \\
\hline
\end{tabular}

Frail, CFS 5-9; non-frail, CFS 1-4.

${ }^{*} \mathrm{P}$ value for comparison between frail and non-frail from log-rank test.

CFS, Clinical Frailty Scale; SE, systemic embolism.

\section{DISCUSSION}

In this study, frailty, hypoalbuminaemia, female sex and malignancy were associated with a higher incidence rate of the composite end points, which included all-cause mortality, stroke or SE, and/or major bleeding. The incidence rate of the composite end points was high and many patients had events shortly after DOAC administration (the median number of months before the occurrence of an adverse event was 5.5 (IQR: 1.8-11.5) months in all patients, and 3.6 (IQR: 1.3-9.3) months in the frail patient group).

In a previous cohort study of Japanese patients with $\mathrm{AF}$, the incidence rate of adverse events (death or stroke/SE) was 8.7 per 100 person-years. ${ }^{17}$ In addition, when the previous study focused on patients weighing less than $50 \mathrm{~kg}$, the incidence rate of adverse events increased to 16.1 per 100 person-years. ${ }^{17}$ In our study, $50 \%$ of patients were frail and their mean body weight was $52.5 \mathrm{~kg}$, so the incidence rate observed in this study was not surprising. Furthermore, the incidence rate of non-cardiovascular events was also consistent with past reports (eg, cardiac death in 1.1 per 100 person-years, non-cardiac death in 5.7 per 100 person-years ${ }^{17}$ and $66 \%$ of patients dying due to non-cardiovascular events ${ }^{18}$ ). Although the median $\mathrm{CHA}_{2} \mathrm{DS}_{2}$-VASc score in the frail group was 5 and the incidence of stroke or other thromboembolic events was expected to be $6.7 \%$ per year, ${ }^{12}$ ischaemic stroke occurred in $6.2 \%$ of frail patients. In addition, although the median HAS-BLED score in the frail group was 2 and the incidence of major bleeding was expected to be $1.88 \%$ per year, ${ }^{19}$ major bleeding occurred in $8.3 \%$ of frail patients. Therefore, it is possible that death and bleeding act as competing events against the decrease in incidence of cerebral infarction in our study. In the frail group, we found that non-cardiac death 


\section{Original research}

Table 3 Indicators of Stroke/Systolic Embolism/Major Bleeding/All-Cause Mortality

\begin{tabular}{|c|c|c|c|c|c|c|}
\hline \multirow[b]{2}{*}{ Factor } & \multicolumn{3}{|c|}{ Univariate } & \multicolumn{3}{|c|}{ Multivariate } \\
\hline & HR & $95 \% \mathrm{Cl}$ & $P$ value & HR & $95 \% \mathrm{Cl}$ & $P$ value \\
\hline Age & 1.08 & 1.04 to 1.13 & $<0.001$ & - & - & - \\
\hline Female & 3.36 & 1.71 to 6.62 & $<0.001$ & 3.49 & 1.73 to 7.07 & 0.001 \\
\hline Weight & 0.96 & 0.93 to 0.99 & 0.004 & - & - & - \\
\hline Hypertension & 2.00 & 0.94 to 4.24 & 0.067 & - & - & - \\
\hline Diabetes mellitus & 0.67 & 0.26 to 1.73 & 0.408 & - & - & - \\
\hline Heart failure & 2.25 & 1.17 to 4.33 & 0.012 & - & - & - \\
\hline Stroke/TIA & 1.43 & 0.75 to 2.73 & 0.274 & - & - & - \\
\hline Bleeding history & 1.93 & 0.59 to 6.30 & 0.270 & - & - & - \\
\hline eGFR & 0.99 & 0.98 to 1.01 & 0.348 & - & - & - \\
\hline $\mathrm{Hb}$ & 0.73 & 0.62 to 0.86 & $<0.001$ & - & - & - \\
\hline Albumin & 0.40 & 0.26 to 0.63 & $<0.001$ & 0.47 & 0.28 to 0.79 & 0.005 \\
\hline Frail & 5.72 & 2.60 to 12.6 & $<0.001$ & 3.71 & 1.59 to 8.65 & 0.002 \\
\hline Malignancy & 3.37 & 1.59 to 7.16 & 0.001 & 4.02 & 1.83 to 8.84 & 0.001 \\
\hline Under-dosed & 2.46 & 0.74 to 8.23 & 0.130 & - & - & - \\
\hline Over-dosed & 0.57 & 0.08 to 4.16 & 0.574 & - & - & - \\
\hline $\mathrm{CHA}_{2} \mathrm{DS}_{2}$-VASc score & 1.41 & 1.17 to 1.71 & $<0.001$ & - & - & - \\
\hline HAS-BLED score & 1.57 & 1.12 to 2.20 & 0.009 & - & - & - \\
\hline
\end{tabular}

Frail: CFS score of 5-9.

$\mathrm{Hb}$, haemoglobin; TIA, transient ischaemic attack; eGFR, estimated glomerular filtration rate.

increased significantly compared with the non-frail group. In past reports, mortality due to undetermined cause increased substantially in accordance with age, and non-cardiovascular death was most strongly associated with anaemia. ${ }^{20}$ This report may indicate that more careful follow-up of major bleeding events is necessary after DOAC administration in older patients.

Frailty is a complex syndrome of decreased stress tolerance that arises from impairments in multiple organ systems, and it is considered a factor in the difference between biological and chronological age. ${ }^{9}$ In general, frailty leads to a higher risk of disability, hospital admission and death, ${ }^{21}$ and this higher risk has been reported in patients with $\mathrm{AF} .{ }^{11}$ In this study, it is interesting that frailty remained a risk factor for the composite end points despite accounting for established risk factors such as age,

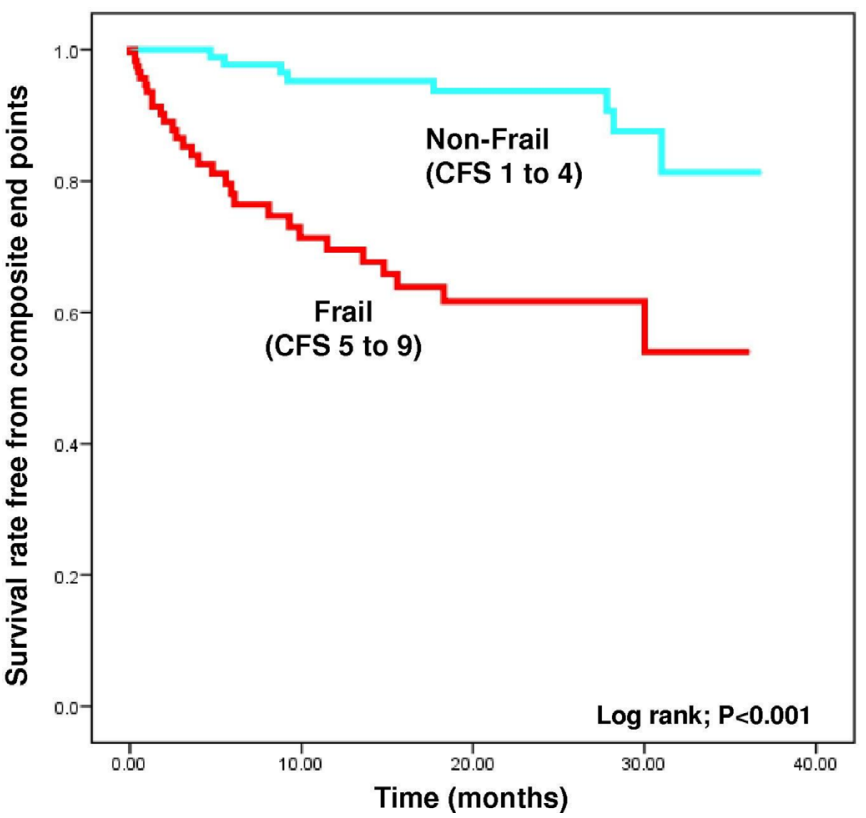

Figure 2 Event-free survival curve for stroke/systemic embolism/major bleeding according to frailty status. CFS, Clinical Frailty Scale
$\mathrm{CHA}_{2} \mathrm{DS}_{2}$-VASc score and HAS-BLED score in the multivariate analysis. On the contrary, there is also a report that AF is associated with frailty, ${ }^{10}$ and it is possible that stress tolerance had become considerably low in these frail patients at the time of AF development. Therefore, frailty could be a strong indicator of these adverse events in patients with AF.

Previous studies have reported that hypoalbuminaemia is an important marker of clinical adverse events such as major bleeding and death, ${ }^{22}$ and our study had similar results. Hypoalbuminaemia has several aetiologies that include decreased synthesis in the liver, lack of intake, systemic inflammation and loss of albumin (nephritic syndrome or protein-losing enteropathy). ${ }^{23}$ However, a detailed pathophysiological mechanism has not been proposed to explain the association between hypoalbuminaemia and clinical events. An association between hypoalbuminaemia and frailty has also been reported. ${ }^{24}$ However, the pathophysiology underlying the relationship between hypoalbuminaemia and frailty is also unclear. The presence of a third factor that can affect both hypoalbuminaemia and frailty has been suggested and includes a decrease in muscle mass, ${ }^{25}$ poor dietary intake and chronic inflammation. ${ }^{26}$ In our study, we did not measure muscle mass, dietary intake or inflammatory markers, so we could not determine the detailed relationship between hypoalbuminaemia and frailty.

Past reports have also associated with anaemia with frailty, ${ }^{27}$ and anaemia has been associated with clinical adverse events in patients with $\mathrm{AF}^{28}$ In this study, univariate analysis showed that anaemia was significantly associated with the composite end points, but the multivariate analysis did not show this significant association. Therefore, it may be clinically important to comprehensively understand the patient's condition instead of only evaluating haemoglobin levels. In patients not eligible for randomised controlled trials after starting DOAC, more careful follow-up is necessary, and a more comprehensive approach is required. ${ }^{9}$

Contrary to existing evidence, stroke or TIA and bleeding history were not predictive of composite end points in the univariate analysis in our study. Bleeding history was an indicator 
of major bleeding, but not stroke or SE. ${ }^{7}$ This could explain why major bleeding was not a predictor of composite end points. In our study, bleeding history was a predictor of major bleeding in the univariate analysis (HR: 4.70, 95\% CI: 1.00 to 22.1). Stroke or TIA history is also a predictor of stroke, but not a significant predictor of major bleeding. ${ }^{7}$ Moreover, our hospital actively performs emergent endovascular treatment of acute ischaemic stroke (about 60 cases a year). These factors may explain why stroke or TIA history was not predictive of composite end points in our study.

Our study has some limitations. First, this study had a small sample size. Second, this study was a retrospective single-centre study, and we could not determine causality. Further studies are needed to address whether improvement in frailty leads to a reduced risk of adverse events in patients with AF. Third, there are several other methods that may be used to evaluate frailty status. ${ }^{29}$ In clinical practice, CFS is superior in its simplicity, and several studies have used this scale score as a prognostic factor for adverse events. ${ }^{30}$ Fourth, follow-up periods were short. Fifth, patients who did not receive DOAC were not studied.

\section{CONCLUSIONS}

Frailty, hypoalbuminaemia, female sex and malignancy were associated with clinical adverse events in patients with AF who were prescribed DOAC.

Funding The authors have not declared a specific grant for this research from any funding agency in the public, commercial or not-for-profit sectors.

Competing interests None declared.

Patient consent for publication Not required.

Provenance and peer review Not commissioned; externally peer reviewed.

Data availability statement All data relevant to the study are included in the article or uploaded as supplementary information.

\section{REFERENCES}

1. Chugh SS, Havmoeller R, Narayanan K, et al. Worldwide epidemiology of atrial fibrillation: a global burden of disease 2010 study. Circulation 2014;129:837-47.

2. Lim CW, Kasim S, Ismail JR, et al. Prevalence of atrial fibrillation in the Malaysian communities. Heart Asia 2016;8:62-6.

3. Wolf PA, Abbott RD, Kannel WB. Atrial fibrillation as an independent risk factor for stroke: the Framingham study. Stroke 1991;22:983-8.

4. Giugliano RP, Ruff CT, Braunwald E, et al. Edoxaban versus warfarin in patients with atrial fibrillation. N Engl J Med 2013;369:2093-104.

5. Granger $\mathrm{CB}$, Alexander JH, McMurray JJV, et al. Apixaban versus warfarin in patients with atrial fibrillation. N Engl J Med 2011;365:981-92.

6. Kato ET, Giugliano RP, Ruff CT, et al. Efficacy and safety of edoxaban in elderly patients with atrial fibrillation in the engage AF-TIMI 48 trial. J Am Heart Assoc 2016;5.

7. Yamashita Y, Uozumi R, Hamatani Y, et al. Current status and outcomes of direct oral anticoagulant use in real-world atrial fibrillation patients - Fushimi AF Registry. Circ J 2017:81:1278-85
8. Yamashita Y, Hamatani Y, Esato M, et al. Clinical Characteristics and Outcomes in Extreme Elderly (Age $\geq 85$ Years) Japanese Patients With Atrial Fibrillation: The Fushimi AF Registry. Chest 2016;149:401-12.

9. Singh $M$, Stewart $R$, White $H$. Importance of frailty in patients with cardiovascular disease. Eur Heart J 2014;35:1726-31.

10. Fumagalli $S$, Tarantini F, Guarducci $L$, et al. Atrial fibrillation is a possible marke of frailty in hospitalized patients: results of the GIFA study. Aging Clin Exp Res 2010;22:129-33.

11. Kim S-W, Yoon S-J, Choi J-Y, et al. Clinical implication of frailty assessment in older patients with atrial fibrillation. Arch Gerontol Geriatr 2017:70:1-7.

12. Lip GYH, Frison L, Halperin JL, et al. Identifying patients at high risk for stroke despite anticoagulation: a comparison of contemporary stroke risk stratification schemes in an anticoagulated atrial fibrillation cohort. Stroke 2010;41:2731-8.

13. D'Agostino RB, Wolf PA, Belanger AJ, et al. Stroke risk profile: adjustment for antihypertensive medication. The Framingham study. Stroke 1994;25:40-3.

14. Matsuo S, Imai E, Horio M, et al. Revised equations for estimated GFR from serum creatinine in Japan. Am J Kidney Dis 2009:53:982-92.

15. Rockwood K, Song X, MacKnight $C$, et al. A global clinical measure of fitness and frailty in elderly people. CMAJ 2005;173:489-95.

16. Schulman S, Kearon C, Subcommittee on Control of Anticoagulation of the Scientific and Standardization Committee of the International Society on Thrombosis and Haemostasis, et al. Definition of major bleeding in clinical investigations of antihemostatic medicinal products in non-surgical patients. J Thromb Haemost 2005;3:692-4

17. Hamatani Y, Ogawa H, Uozumi R, et al. Low Body Weight Is Associated With the Incidence of Stroke in Atrial Fibrillation Patients - Insight From the Fushimi AF Registry. Circ J 2015;79:1009-17.

18. Fauchier L, Samson A, Chaize G, et al. Cause of death in patients with atrial fibrillation admitted to French hospitals in 2012: a nationwide database study. Open Heart 2015:2:e000290.

19. Pisters R, Lane DA, Nieuwlaat $R$, et al. A novel user-friendly score (HAS-BLED) to assess 1-year risk of major bleeding in patients with atrial fibrillation: the Euro Heart Survey. Chest 2010;138:1093-100.

20. An Y, Ogawa H, Yamashita Y, et al. Causes of death in Japanese patients with atrial fibrillation: the Fushimi atrial fibrillation registry. Eur Heart J Qual Care Clin Outcomes 2019;5:35-42.

21. Wallis SJ, Wall J, Biram RWS, et al. Association of the clinical frailty scale with hospital outcomes. QJM 2015;108:943-9.

22. Corona LP, de Oliveira Duarte YA, Lebrão ML. Markers of nutritional status and mortality in older adults: the role of anemia and hypoalbuminemia. Geriatr Gerontol Int 2018;18:177-82.

23. Kim S, McClave SA, Martindale RG, et al. Hypoalbuminemia and clinical outcomes: what is the mechanism behind the relationship? Am Surg 2017:83:1220-7.

24. Okamura T, Hayakawa T, Hozawa A, et al. Lower levels of serum albumin and total cholesterol associated with decline in activities of daily living and excess mortality in a 12-year cohort study of elderly Japanese. J Am Geriatr Soc 2008;56:529-35.

25. Visser M, Kritchevsky SB, Newman AB, et al. Lower serum albumin concentration and change in muscle mass: the health, aging and body composition study. Am J Clin Nutr 2005;82:531-7.

26. Fontana L, Addante F, Copetti $M$, et al. Identification of a metabolic signature for multidimensional impairment and mortality risk in hospitalized older patients. Aging Cell 2013;12:459-66

27. Roy CN. Anemia in frailty. Clin Geriatr Med 2011;27:67-78.

28. Sharma S, Gage BF, Deych E, et al. Anemia: an independent predictor of death and hospitalizations among elderly patients with atrial fibrillation. Am Heart J 2009;157:1057-63.

29. Rockwood K, Mitnitski A. Frailty defined by deficit accumulation and geriatric medicine defined by frailty. Clin Geriatr Med 2011:27:17-26.

30. Shimura T, Yamamoto $M, K a n o ~ S$, et al. Impact of the clinical frailty scale on outcomes after transcatheter aortic valve replacement. Circulation 2017:135:2013-24. 